\title{
Dual Tasking Under Compromised Visual and Somatosensory Input in Elderly Fallers and Non-Fallers
}

\author{
Jaap Swanenburg ${ }^{*, 1}$, Eling D. de Bruin ${ }^{2}$, Stefan Hegemann ${ }^{3}$, Daniel Uebelhart ${ }^{1,4}$ and Theo Mulder ${ }^{5,6}$ \\ ${ }^{I}$ Department of Rheumatology and Institute of Physical Medicine, University Hospital Zurich, Zurich, Switzerland \\ ${ }^{2}$ Institute of Human Movement Sciences and Sport, ETH, Zurich, Switzerland \\ ${ }^{3}$ Department of Otorhinolaryngology, Head and Neck Surgery, University Hospital Zurich, and Zurich Center for \\ Integrative Human Physiology, Zurich, Switzerland \\ ${ }^{4}$ Centre of Osteoporosis, University Hospital Zurich, Zurich, Switzerland \\ ${ }^{5}$ Royal Netherlands Academy of Arts and Sciences, Amsterdam, The Netherlands \\ ${ }^{6}$ Center for Human Movement Sciences, University Medical Center Groningen, Groningen, The Netherlands
}

\begin{abstract}
Background: Performance of additional tasks disturbs postural control in elderly. It is unknown, however, how postural control is affected in elderly fallers and non-fallers in a reduced sensory situation.

Objective: To compare differences between single and dual tasking in three test conditions; (1) no-vision, (2) under reduced somatosensory information and (3) with a combination of both conditions.

Design: An observational cohort study with participants assigned to a 12-month pretest fall assessment and a postural balance assessment.

Methods: Fifteen independently living elderly participated (77.5 \pm 7.0 [63-87] years). Falls were pre-assessed with a 1year monthy "fall calendar". Postural control was analyzed by means of a force platform. Participants were standing quiet (first task) while counting backwards (second task). A 2-factor (group x condition) ANOVA was performed at $p<.05$. Differences of postural (DTCp) and cognitive dual task costs (DTCc) between test conditions were analyzed (one-way ANOVA).

Results: The analysis showed significant group (fallers/non-fallers) and condition effects. Post hoc analyses indicated that the postural control variables were significantly different during the concurrent reduced vision and somatosensory information.

Dual task costs showed a significant difference between normal $(\mathrm{N})$ and the combined condition $(\mathrm{NV}+\mathrm{RP})$ in non-fallers.

Conclusion: The combination of reduced visual and somatosensory information causes a larger disturbance of postural stability compared with the reduction of visual or somatosensory information alone. Non-fallers seem to have no threats to the postural control stability in this combined reduced sensory situation. They reduce their postural control, which leaves them enough resources to compensate for the reduced sensory information.
\end{abstract}

Keywords: Postural control, dual task, fallers, vision, somatosensoric.

\section{BACKGROUND}

The accurate processing of sensory information is critical for maintaining balance within the variety of environmental situations encountered in daily life. Many elderly persons have proprioceptive deficits in position and motion sensing and these age-related declines may compromise sensorimotor tasks such as balance and postural control. These changes are most likely attributable to degenerative changes in the peripheral nervous system as well as to decreases in central processing abilities [1]. The effects of

*Address correspondence to this author at the Department of Rheumatology and Institute of Physical Medicine, University Hospital Zurich, Gloriastrasse 25, CH-8091 Zurich, Switzerland; Tel: +41 (0) 4425524 91; Fax: +41 (0) 4425543 88; E-mail: jaap.swanenburg@usz.ch aging caused by deterioration of the sensory system can be tested by artificially reducing sensory input, e.g., somatosensory, vestibular, and/or visual inputs. This artificial reduction disturbs postural control $[2,3]$.

Under normal circumstances in healthy adults, dual tasking does not pose a challenge for everyday life functioning [4], but performing two tasks simultaneously may become a relevant problem in older adults [5-7]. Indeed, the simultaneous performance of an attention-demanding task and a postural task may result in decreased postural control [5], such as can be observed in the "stops walking while talking" phenomenon described by Lundin-Olsson et al. [6]. In the worst case this decreased postural stability may result in a fall [8]. Since injuries resulting from falls are one of the leading causes of death among the elderly population 
[9], it is important to be able to discriminate between (potential) fallers and non-fallers.

When older adults are tested for dual-task performance while standing on a stable surface, they exhibit an increase in postural instability compared with the single-task condition [8]. The results of Doumas et al. suggest that when posture is relatively stable, older adults have the flexibility to allow additional instability to release the resources necessary to accommodate the demands of dual task performance. Doumas and colleagues also showed that only somatosensory manipulation led to pronounced age-effects in posture. A similar effect due to manipulation of vision was not seen [8].

It is unclear, however, what the effects of a combined manipulation, e.g., reduced vision and reduced somatosensory input, on postural stability are. It can be speculated that the combined manipulation will further worsen postural control in order to accommodate accurate cognitive task performance [9]. It is also possible, however, that during a combined reduction of both visual and somatosensory input, postural control will be prioritized at the cost of cognitive task performance.

The results of studies investigating the relationship between postural control and memory performance under conditions where visual and somatosensory input have been manipulated can be interpreted in terms of two main theories. The first theory, the theoretical framework of resource-sharing [10], states that concurrent tasks interfere with each other due to simultaneous demands on a capacitylimited pool of resources. So, if two tasks are performed concurrently, performance on one or both may deteriorate if they require more than the available attentional resources $[10,11]$.

The second theory, the adaptive resource-sharing model [9] maintains that various factors play part in observed increases and decreases in sway across experimental conditions. This model stresses that the primary functions of the postural control system are to stabilize balance and facilitate supra-postural task performance. In the absence of threats to stability postural control has a facilitatory function for the performance of supra-postural tasks. Facilitation occurs either through increases or decreases in postural sway, depending on the nature of the task [9].

To determine which of these two theories should be preferred we designed an experimental set-up in which we tested postural control of elderly fallers and non-fallers under different manipulations. This pilot study aimed to compare differences between single and dual tasking in three test conditions; (1) no-vision, (2) under reduced somatosensory information and (3) with a combination of both conditions.

\section{METHODS}

\section{Participants}

This study was conducted at the University Hospital Zurich, Zurich, Switzerland. Participants were recruited within the hospital patient population by word of mouth. Fifteen elderly living in the community were consecutively recruited at the Department of Rheumatology and the Institute of Physical Medicine.
The mean age of the participants was $77.5 \pm 7$ years (range, 63-87 years). The inclusion criteria were age 60 years or older of either gender. The exclusion criteria were inability to understand the German language and/or the purpose of the study, severe psychological or psychiatric problems, chronic substance abuse (e.g., medication or alcohol), and/or undergoing therapy with neuroleptics, sedatives, antiepileptics, or antidepressants. All participants provided written informed consent. The study was approved and registered by the local ethical committee of Canton Zurich, Switzerland, under SPUK EK 649.

\section{Study Design}

This study was an observational cohort study. The design of the study consisted of a 12-month pretest fall assessment and a postural balance assessment as follow-up. This procedure was chosen to rule out possible effects of balance assessment on fall behavior. The participant recorded falls for 1 year with a monthly fall calendar. Thereafter, the postural balance of the participant was tested within a single assessment session that lasted approximately 40 minutes. The postural balance measurements were collected under two task situations: standing quiet (single task) and standing quiet combined with counting backwards in steps of seven (dual task). Both the single- and dual-task test situations were performed in following conditions; [1] standing quiet (N), no sensory manipulation [2], standing quiet without vision (NV), [3] standing quiet with reduced somatosensory information (RS) and [4] standing quiet with a combination of no-vision and reduced somatosensory information $(\mathrm{NV}+\mathrm{RS})$. Before the measurement started, instructions for the cognitive task were given followed by performance of the counting backward task while seated. Between each task, the participants were allowed to sit for a 2-minute break. The order of tasks was changed randomly to control for fatigue and learning effects. The measurer was blinded to the fall status.

\section{Single and Dual Task}

For the single task (standing quiet), the participants were instructed to stand comfortably in a preferred stable, double-leg stance position on the force platform with the arms hanging by the sides and eyes open while looking straight ahead. Because a change in the base of support (BOS) has a substantial effect on postural control [5], the outlines of both feet were marked on the platform to obtain standardized foot positions across the successive measurements for each individual participant. Counting backwards was previously shown to cause a significant degradation in postural stability in healthy elderly [12-14] and was, therefore, chosen as the attention-demanding (cognitive) secondary task. The participant was asked to count backwards as fast and accurate as possible in 20 seconds [15, 16]. The starting number was selected at random from a range of 80 to 99. The counting was controlled continuously for accuracy and every mistake was noted. The results of the cognitive performance were defined in terms of the correct answers minus the mistakes made. No feedback on performance was given during the testing to avoid any extra performance pressure.

\section{Test Conditions}

The participants were instructed to view a fixed horizontal grey cross $(1 \mathrm{~m} \times 0.5 \mathrm{~m})$ in the middle of a screen 
$(1.5 \mathrm{~m} \times 1.5 \mathrm{~m})$ positioned $2 \mathrm{~m}$ in front of the forceplate. The height of the gray cross was at $1.5 \mathrm{~m}$. All participants used their own glasses when needed to have optimal individual visual acuity. In the no vision condition, vision was occluded with a pair of custom-made opaque goggles that prevented the subject from perceiving visual information without blocking the light in general. The participants were instructed to keep their eyes open inside the goggles. In the reduced somatosensory condition (RS), the participant took a barefooted, double-legged stance on a foam layer in the standardized foot position. The foam was $10 \mathrm{~cm} \times 50 \mathrm{~cm} \times$ $50 \mathrm{~cm}$ (height $\times$ length $\times$ width) and had a medium density of $45.2 \mathrm{~kg} / \mathrm{m}^{3}$. Participants did not have direct contact to the forceplate.

\section{Posturography}

An AMTI Accusway system (Advanced Mechanical Technology, Inc., Watertown, MA), consisting of a portable force platform and SWAYWIN software for data acquisition and analysis, was used for data collection. This system measures ground-reacting force and moments in three orthogonal directions $(50-\mathrm{Hz}$ sampling frequency). These measurements provide the centre-of-pressure coordinates, which enable the calculation of the maximum displacement in the anteroposterior and medial-lateral directions (Max-AP, Max-ML), mean displacement in the medial-lateral direction (MML), the root mean square amplitude in antero-posterior and medial-lateral directions (RMS-AP, RMS-ML), the average speed of displacement (V), and the area of the 95th percentile ellipse (AoE) with good reliability [16].

\section{Falls}

The participants were asked to take a 1-year "fall calendar" home and were expected to document fall events monthly. After each month, a calendar page (with a stamped, pre-addressed envelope) was expected to be sent to the investigators. Participants who did not return the calendar page at the end of the month were contacted by telephone. A fall was defined as any event leading to unintentionally coming to the ground or some lower level and other than as a consequence of sustaining a violent blow, loss of consciousness, sudden onset of paralysis like in stroke, or epileptic seizure [17]. Neither "coming to rest against a wall or other structure" nor "high-trauma falls (e.g., falling from a ladder) and "falling as a consequence of sustaining a violent blow" were included as falls in this study [18]. Two groups were defined after 12 months of fall assessment; [1] fallers endured two or more falls and [2] non-fallers zero or one fall.

\section{Fall Risk Factors}

The participants were interviewed about medical conditions such as dizziness, low blood pressure, visual impairments, muscle weakness, osteoporosis, foot problems, as well as daily alcohol consumption (two or more beverages per day). Furthermore, the use of drugs like benzodiazepines, psychotropics, class 1a antiarrhythmic medications, digoxin, diuretics, and sedatives and/or the use of more than four different medications (multiple medicine use) were noted. Participants were also asked whether they felt unsafe while standing and/or walking and about their fear of falling. Participants who obtained groceries by foot or participated in any other sports or physical activity were not scored as being sedentary in behaviour. These variables were dichotomised as 0 (absent) or 1 (present).

\section{Data Analysis}

Descriptive statistics was used to describe the participant's demographic variables. The four measurements of each task were averaged to obtain a reliable measure [1619]. The one-sample Kolmogorov-Smirnov test was used to check the normality of the distributions. A linear regression model was used to control for confounding factors regarding fallers and included age, body mass index, gender and all fall risk factors. After adjusting for potential confounders, an 8 (test conditions) $\times 2$ (fallers/non-fallers) fractional analysis of variance (ANOVA) was conducted. Significance for the analysis was set at $p<.05$. The magnitude of statistical test condition differences was expressed in terms of Cohens dstatistic. An effect size of 0.20 was considered small, 0.50 moderate and 0.80 large [20]. Bonferroni post hoc analysis was performed to test for differences between the different test conditions when the first stage of analysis revealed significant effects. Dual-task costs (DTC) express the effects of additional costs imposed in individual task performance in a dual-task setting [8]. The DTC were calculated for posture (DTCp) and for cognition (DTCc). The formula used for the DTCp is: [(dual-task - single-task)/single-task] $\times 100$. Positive DTCp are reflected in an increase in postural instability. The formula used for the DTCc is: [(single-task dual-task) $/$ single-task] $\times 100$. Positive DTCc are reflected in a decrease in counting performance $[8,21]$. DTCc is tested with a 4 (conditions $\mathrm{N}, \mathrm{NV}, \mathrm{RP}, \mathrm{NV}+\mathrm{RP}) \times 2$ (fallers/nonfallers) fractional analysis of variance (ANOVA). Differences of DTCp between the test conditions $(\mathrm{N}, \mathrm{NV}$, $\mathrm{RP}, \mathrm{NV}+\mathrm{RP}$ ) in all, fallers and non-fallers were analyzed with a one-way ANOVA. The data were entered, stored, and analysed in SPSS 15.0 statistical software (SPSS, Inc., Chicago, IL).

\section{RESULTS}

\section{Participants}

All 15 elderly persons who participated in this study completed 12 consecutive monthly fall calendars and were able to perform the test conditions. Table 1 shows all fall risk factors.

The mean age was 77.5 (standard deviation, 7) years. The participants' characteristics are summarized in Table 2. The 12 monthly calendar pages revealed a total of six fallers. In total, 23 falls were recorded. Six falls in six subjects resulted in a fall-related injury, five contusions and one sprain. The body mass index was found to be a confounder and, therefore, adjusted for. Counting strategy and sum of counting mistakes made are summarized in Table 2. The mean values of all variables in all conditions distributed in fallers and non-fallers are summarised in Table 3. Fig. (1) shows the RMS-ML variable in all test conditions and for the distribution of the sample in fallers and non-fallers.

\section{Main Effects Between Single- and Dual-Task}

The factorial ANOVA revealed main significant effects between the single- and dual-task situations in balance 
Table 1. Fall Risk Factors

\begin{tabular}{|c|c|c|c|}
\hline Fall Risk Factors \% & All $(n=15)$ & Fallers $(n=6)$ & Non-Fallers $(\mathbf{n}=\mathbf{9})$ \\
\hline \multicolumn{4}{|l|}{ Drugs } \\
\hline Risk factor medications or more than four medications (multiple medicine use) & 9 & 5 & 4 \\
\hline \multicolumn{4}{|l|}{ Medical conditions } \\
\hline Dizziness & 5 & 3 & 2 \\
\hline Low blood pressure & 1 & 0 & 1 \\
\hline \multicolumn{4}{|l|}{ Sedentary behavior } \\
\hline Less active & 3 & 3 & 0 \\
\hline Muscle weakness & 3 & 2 & 1 \\
\hline \multicolumn{4}{|l|}{ Psychologic status } \\
\hline Fear of falling & 2 & 1 & 1 \\
\hline \multicolumn{4}{|l|}{ Nutritional deficiencies } \\
\hline Low body mass index $\left(<18.5 \mathrm{~kg} / \mathrm{m}^{2}\right)$ & 0 & 0 & 0 \\
\hline Osteoporosis & 1 & 1 & 0 \\
\hline Foot pain & 0 & 0 & 0 \\
\hline
\end{tabular}

Table 2. Baseline Characteristics of the Participants

\begin{tabular}{|c|c|c|c|}
\hline & All & Fallers & Non-Fallers \\
\hline Female & 8 & 3 & 5 \\
\hline Male & 7 & 3 & 4 \\
\hline Age (years \pm SD) & $77.5 \pm 7$ & $83 \pm 6$ & $77.2 \pm 7$ \\
\hline Age range & $63 / 87$ & $70 / 95$ & $63 / 87$ \\
\hline Weight $(\mathrm{kg} \pm \mathrm{SD})$ & $70.9 \pm 16$ & $66 \pm 11$ & $74.7 \pm 11$ \\
\hline Length $(\mathrm{cm} \pm \mathrm{SD})$ & $167.3 \pm 9$ & $163 \pm 8$ & $169 \pm 8$ \\
\hline Body mass index $\left(\mathrm{kg} / \mathrm{m}^{2} \pm \mathrm{SD}\right)$ & $24.9 \pm 5$ & $23.5 \pm 5$ & $26.0 \pm 2$ \\
\hline \multicolumn{4}{|l|}{ Mental task } \\
\hline Serial 7s (average) & 15 & 6 & 9 \\
\hline Serial 3s & 0 & 0 & 0 \\
\hline \multicolumn{4}{|l|}{ Counting mistakes } \\
\hline Normal (Sum) & 19 & 7 & 12 \\
\hline No vision & 19 & 8 & 11 \\
\hline Reduced proprioception & 14 & 4 & 10 \\
\hline No vision/reduced proprioception & 20 & 10 & 10 \\
\hline Average per participant & 1.2 & 1.2 & 1.2 \\
\hline
\end{tabular}

SD, standard deviation; BOS, base of support.

variables: Max-ML $(\mathrm{F}[7 / 104]=19.506, \mathrm{p}<0.001)$, RMS$\operatorname{ML}(\mathrm{F}[7 / 104]=21.855, \mathrm{p}<0.000)$, Max-AP $(\mathrm{F}[7 / 104]=$ $17.075, \mathrm{p}<0.001)$, RMS-AP $(\mathrm{F}[7 / 104]=16.027, \mathrm{p}<0.001)$, $\mathrm{V}(\mathrm{F}[7 / 104]=10.789, \mathrm{p}<0.001)$, and $\mathrm{AoE}(\mathrm{F}[7 / 104]=$ $16.334, \mathrm{p}<0.001)$. There was a moderate magnitude of statistically significant group differences (effect size between 0.5 and 0.6). Bonferroni post-hoc analysis showed that dual tasking within each condition had no significant effect (see Table 4). Bonferroni post hoc analysis split for fallers and non-fallers showed the same results for the test situations.

\section{Main Effects Between Fallers and Non-Fallers}

Significant effects were found in postural balance variables: Max-ML $(\mathrm{F}[1 / 104]=6.618, p=0.012)$, RMS-ML $(\mathrm{F}[1 / 104]=10.802, p=0.001), \operatorname{Max}-\mathrm{AP}(\mathrm{F}[1 / 104]=6.735$, $p=0.011)$, RMS-AP $(\mathrm{F}[1 / 104]=9.587, p=0.003)$, and AoE $(\mathrm{F}[1 / 104]=4.276, p=0.041)$. No significant main effect between multiple fallers and non-fallers was found in $\mathrm{V}$ $(\mathrm{F}[1 / 104]=0.020, p=0.888)$. The magnitude of both 
Table 3. Means of All Variables in All Conditions

\begin{tabular}{|c|c|c|c|c|c|c|c|c|}
\hline & $\begin{array}{c}\mathbf{N} \\
\text { Mean }\end{array}$ & $\begin{array}{c}\text { DT } \\
\text { Mean }\end{array}$ & $\begin{array}{c}\text { NV } \\
\text { Mean }\end{array}$ & $\begin{array}{c}\text { DT+NV } \\
\text { Mean }\end{array}$ & $\begin{array}{c}\text { RP } \\
\text { Mean }\end{array}$ & $\begin{array}{l}\text { DT+RP } \\
\text { Mean }\end{array}$ & $\begin{array}{c}\text { NV+RP } \\
\text { Mean }\end{array}$ & $\begin{array}{c}\mathrm{DT}+\mathrm{NV}+\mathrm{RP} \\
\quad \text { Mean }\end{array}$ \\
\hline \multicolumn{9}{|l|}{ Max-ML(SD) } \\
\hline all & $0.65(0.3)$ & $1.02(0.5)$ & $1.03(0.9)$ & $1.10(0.5)$ & $2.00(0.8)$ & $2.40(1.1)$ & $2.95(1.2)$ & $3.40(1.6)$ \\
\hline Fallers & $0.61(0.3)$ & $0.87(0.5)$ & $1.29(1.4)$ & $1.05(0.5)$ & $2.39(1.0)$ & $3.09(1.3)$ & $3.16(1.1)$ & $4.19(1.9)$ \\
\hline Non-Fallers & $0.68(0.4)$ & $1.12(0.5)$ & $0.84(0.4)$ & $1.14(0.5)$ & $1.73(0.6)$ & $1.93(0.6)$ & $2.81(1.3)$ & $2.88(1.2)$ \\
\hline \multicolumn{9}{|l|}{ RMS-ML (SD) } \\
\hline all & $0.26(0.1)$ & $0.39(0.2)$ & $0.38(0.3)$ & $0.42(0.2)$ & $0.74(0.3)$ & $0.92(0.4)$ & $1.23(0.5)$ & $1.45(0.7)$ \\
\hline Fallers & $0.25(0.1)$ & $0.35(0.2)$ & $0.47(0.4)$ & $0.42(0.3)$ & $0.94(0.5)$ & $1.19(0.5)$ & $1.46(0.7)$ & $1.84(0.9)$ \\
\hline Non-Fallers & $0.28(0.1)$ & $0.42(0.2)$ & $0.32(0.1)$ & $0.41(0.2)$ & $0.62(0.2)$ & $0.73(0.3)$ & $1.08(0.4)$ & $1.19(0.5)$ \\
\hline \multicolumn{9}{|l|}{ Max-AP (SD) } \\
\hline all & $1.15(0.5)$ & $1.52(0.7)$ & $1.51(0.8)$ & $1.88(0.9)$ & $2.02(0.7)$ & $2.44(0.9)$ & $3.58(1.3)$ & $3.49(1.0)$ \\
\hline Fallers & $1.12(0.6)$ & $1.44(0.7)$ & $1.76(1.0)$ & $1.99(1.0)$ & $2.49(1.2)$ & $2.81(1.0)$ & $4.11(1.6)$ & $3.87(1.0)$ \\
\hline Non-Fallers & $1.09(0.5)$ & $1.58(0.8)$ & $1.35(0.6)$ & $1.82(0.8)$ & $1.73(0.4)$ & $2.20(0.7)$ & $3.23(0.9)$ & $3.23(1.0)$ \\
\hline \multicolumn{9}{|l|}{ RMS-AP (SD) } \\
\hline all & $0.45(0.2)$ & $0.59(0.3)$ & $0.61(0.3)$ & $0.71(0.3)$ & $0.79(0.4)$ & $0.89(0.3)$ & $1.43(0.5)$ & $1.47(0.5)$ \\
\hline Fallers & $0.44(0.2)$ & $0.59(0.3)$ & $0.71(0.5)$ & $0.78(0.4)$ & $1.07(0.7)$ & $1.09(0.3)$ & $1.60(0.6)$ & $1.69(0.5)$ \\
\hline Non-Fallers & $0.42(0.2)$ & $0.59(0.3)$ & $0.54(0.2)$ & $0.66(0.3)$ & $0.63(0.2)$ & $0.76(0.2)$ & $1.31(0.5)$ & $1.32(0.4)$ \\
\hline \multicolumn{9}{|l|}{$\mathbf{V}(\mathrm{SD})$} \\
\hline all & $2.09(1.1)$ & $2.86(1.9)$ & $3.03(1.9)$ & $3.73(2.6)$ & $3.86(1.1)$ & $4.86(1.8)$ & $6.51(2.8)$ & $7.48(2.8)$ \\
\hline Fallers & $2.24(1.3)$ & $2.39(1.7)$ & $3.27(2.1)$ & $3.59(2.2)$ & $4.23(1.6)$ & $5.44(2.0)$ & $5.61(2.2)$ & $7.81(3.2)$ \\
\hline Non-Fallers & $1.87(1.1)$ & $3.17(2.1)$ & $2.87(0.2)$ & $3.84(3.0)$ & $3.52(1.1)$ & $4.48(1.7)$ & $7.11(3.1)$ & $7.26(2.7)$ \\
\hline \multicolumn{9}{|l|}{ AoE (SD) } \\
\hline all & $2.22(1.6)$ & $5.15(5.0)$ & $5.30(6.5)$ & $6.05(5.1)$ & $10.99(7.2)$ & $16.19(9.4)$ & $36.38(24.9)$ & $41.42(29.8)$ \\
\hline Fallers & $1.80(1.1)$ & $3.64(3.0)$ & $7.22(9.8)$ & $5.64(4.6)$ & $16.13(12.5)$ & $22.58(10.6)$ & $43.50(30.4)$ & $50.74(32.9)$ \\
\hline Non-Fallers & $2.37(1.7)$ & $6.15(5.9)$ & $4.02(2.9)$ & $6.32(5.6)$ & $8.06(3.6)$ & $11.94(5.9)$ & $31.63(21.0)$ & $35.21(27.7)$ \\
\hline
\end{tabular}

$\mathrm{SD}=$ Standard Deviation, N=Normal, NV= No Vision, RP= Reduced Proprioception, NV+RP= No Vision + Reduced Proprioception, DTC, Dual-Task Costs; ML, Medial-Lateral; RMS, root mean square; AP, anterior-posterior; V, average speed of displacement; AoE, area of the 95th percentile ellipse.

statistically significant and non-significant group differences was poor (effect sizes between 0.01 and 0.10 ).

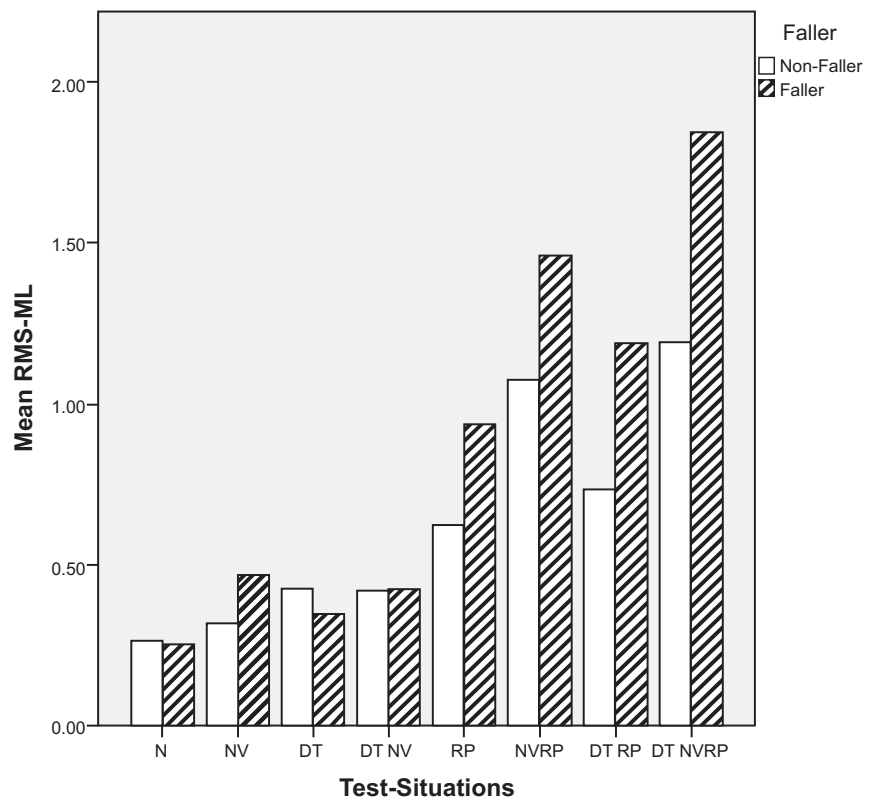

Fig. (1). The RMS-ML variable in all test situations distributed in fallers and non-fallers.

\section{Differences Between Test Conditions}

One-way ANOVA revealed significant differences between the normal condition $(\mathrm{N})$ and the combined somatosensory task (NVRP) for the DTCp. One-way ANOVA showed significant differences between the normal conditions $(\mathrm{N})$ and the combined somatosensory task $(\mathrm{NV}+\mathrm{RP})$ only within the group of non-fallers in DTCp. Results are presented in Table $\mathbf{5}$.

Within the DTCc the one-way ANOVA revealed in all subjects and split-up in fallers and non-fallers (all/fallers/non-fallers) no effects between condition N/NV $(\mathrm{p}=0.962 / 0.371 / 0.378), \mathrm{N} / \mathrm{RP}(\mathrm{p}=0.239 / 0.986 / 0.118)$ and $\mathrm{N} / \mathrm{NV}+\mathrm{RP}(\mathrm{p}=0.542 / 0.498 / 0.862)$.

\section{DISCUSSION}

The different manipulations led to a disturbance of postural control. These findings are not really a surprise and are in concordance with the results of an earlier study from Doumas and colleagues (2008) [8]. However, the added information of this study is that it shows that the combination of manipulations leads to a more marked change in postural control. Adding a cognitive task during the combined reduced sensory situation causes the postural control to be even more worsened. Possibly this reduced postural control was caused to accommodate the facilitation of an accurate cognitive task performance. 
Table 4. Bonferroni Post Hoc Analysis Between All Test Situations and All Variables

\begin{tabular}{|c|c|c|c|c|c|c|c|}
\hline Max-ML & $\mathbf{N}$ & NV & DT & $\mathrm{DT}+\mathrm{NV}$ & $\mathbf{R P}$ & $\mathbf{N V}+\mathbf{R P}$ & $\mathbf{D T}+\mathrm{RP}$ \\
\hline NV & 1.000 & & & & & & \\
\hline DT & 1.000 & 1.000 & & & & & \\
\hline $\mathrm{DT}+\mathrm{NV}$ & 1.000 & 1.000 & 1.000 & & & & \\
\hline $\mathrm{RP}$ & $0.003 *$ & 0.125 & 0.120 & 0.242 & & & \\
\hline $\mathrm{NV}+\mathrm{RP}$ & $<0.001^{*}$ & $<0.001^{*}$ & $<0.001^{*}$ & $<0.001 *$ & 0.147 & & \\
\hline $\mathrm{DT}+\mathrm{RP}$ & $<0.001^{*}$ & $0.002 *$ & $0.002 *$ & $0.005^{*}$ & 1.000 & 1.000 & \\
\hline $\mathrm{DT}+\mathrm{NV}+\mathrm{RP}$ & $<0.001^{*}$ & $<0.001^{*}$ & $<0.001^{*}$ & $<0.001 *$ & $0.002 *$ & 1.000 & 0.091 \\
\hline RMS-ML & $\mathbf{N}$ & NV & DT & $\mathbf{D T}+\mathrm{NV}$ & $\mathbf{R P}$ & $\mathbf{N V}+\mathbf{R P}$ & $\mathrm{DT}+\mathrm{RP}$ \\
\hline NV & 1.000 & & & & & & \\
\hline DT & 1.000 & 1.000 & & & & & \\
\hline $\mathrm{DT}+\mathrm{NV}$ & 1.000 & 1.000 & 1.000 & & & & \\
\hline $\mathrm{RP}$ & $0.019 *$ & 0.250 & 0.387 & 0.558 & & & \\
\hline $\mathrm{NV}+\mathrm{RP}$ & $<0.001^{*}$ & $<0.001^{*}$ & $<0.001^{*}$ & $<0.001 *$ & $0.022 *$ & & \\
\hline $\mathrm{DT}+\mathrm{RP}$ & $<0.001^{*}$ & $0.006^{*}$ & $0.008^{*}$ & $0.016^{*}$ & 1.000 & 0.718 & \\
\hline $\mathrm{DT}+\mathrm{NV}+\mathrm{RP}$ & $<0.001^{*}$ & $<0.001^{*}$ & $<0.001^{*}$ & $<0.001^{*}$ & $<0.001 *$ & 1.000 & $0.005^{*}$ \\
\hline Max-AP & $\mathbf{N}$ & NV & DT & $\mathrm{DT}+\mathrm{NV}$ & RP & $\mathbf{N V + R P}$ & $\mathrm{DT}+\mathrm{RP}$ \\
\hline NV & 1.000 & & & & & & \\
\hline DT & 1.000 & 1.000 & & & & & \\
\hline $\mathrm{DT}+\mathrm{NV}$ & 0.415 & 1.000 & 1.000 & & & & \\
\hline $\mathrm{RP}$ & 0.115 & 1.000 & 1.000 & 1.000 & & & \\
\hline $\mathrm{NV}+\mathrm{RP}$ & $<0.001^{*}$ & $<0.001^{*}$ & $<0.001^{*}$ & $<0.001^{*}$ & $<0.001^{*}$ & & \\
\hline $\mathrm{DT}+\mathrm{RP}$ & $0.001 *$ & 0.117 & 0.129 & 1.000 & 1.000 & $0.014 *$ & \\
\hline $\mathrm{DT}+\mathrm{NV}+\mathrm{RP}$ & $<0.001^{*}$ & $<0.001^{*}$ & $<0.001^{*}$ & $<0.001^{*}$ & $<0.001 *$ & 1.000 & $0.037 *$ \\
\hline RMS-AP & $\mathbf{N}$ & NV & DT & $\mathrm{DT}+\mathrm{NV}$ & RP & NV+RP & $\mathrm{DT}+\mathrm{RP}$ \\
\hline $\mathrm{NV}$ & 1.000 & & & & & & \\
\hline DT & 1.000 & 1.000 & & & & & \\
\hline $\mathrm{DT}+\mathrm{NV}$ & 1.000 & 1.000 & 1.000 & & & & \\
\hline $\mathrm{RP}$ & 0.215 & 1.000 & 1.000 & 1.000 & & & \\
\hline $\mathrm{NV}+\mathrm{RP}$ & $<0.001^{*}$ & $<0.001^{*}$ & $<0.001^{*}$ & $<0.001^{*}$ & $<0.001 *$ & & \\
\hline $\mathrm{DT}+\mathrm{RP}$ & $0.028 *$ & 1.000 & 0.807 & 1.000 & 1.000 & $0.005^{*}$ & \\
\hline $\mathrm{DT}+\mathrm{NV}+\mathrm{RP}$ & $<0.001^{*}$ & $<0.001^{*}$ & $<0.001^{*}$ & $<0.001 *$ & $<0.001 *$ & 1.000 & $0.002 *$ \\
\hline $\mathbf{V}$ & $\mathbf{N}$ & NV & DT & $\mathrm{DT}+\mathrm{NV}$ & $\mathbf{R P}$ & $\mathbf{N V}+\mathbf{R P}$ & $\mathbf{D T}+\mathrm{RP}$ \\
\hline $\mathrm{NV}$ & 1.000 & & & & & & \\
\hline DT & 1.000 & 1.000 & & & & & \\
\hline $\mathrm{DT}+\mathrm{NV}$ & 0.894 & 1.000 & 1.000 & & & & \\
\hline $\mathrm{RP}$ & 0.723 & 1.000 & 1.000 & 1.000 & & & \\
\hline $\mathrm{NV}+\mathrm{RP}$ & $<0.001^{*}$ & $0.001 *$ & $<0.001^{*}$ & $0.019 *$ & $0.025^{*}$ & & \\
\hline $\mathrm{DT}+\mathrm{RP}$ & $0.014 *$ & 0.622 & 0.355 & 1.000 & 1.000 & 1.000 & \\
\hline $\mathrm{DT}+\mathrm{NV}+\mathrm{RP}$ & $<0.001^{*}$ & $<0.001^{*}$ & $<0.001^{*}$ & $<0.001 *$ & $<0.001^{*}$ & 1.000 & $0.035^{*}$ \\
\hline AoE & $\mathbf{N}$ & NV & DT & $\mathbf{D T}+\mathrm{NV}$ & RP & $\mathbf{N V}+\mathbf{R P}$ & $\mathbf{D T}+\mathrm{RP}$ \\
\hline $\mathrm{NV}$ & 1.000 & & & & & & \\
\hline DT & 1.000 & 1.000 & & & & & \\
\hline $\mathrm{DT}+\mathrm{NV}$ & 1.000 & 1.000 & 1.000 & & & & \\
\hline $\mathrm{RP}$ & 1.000 & 1.000 & 1.000 & 1.000 & & & \\
\hline $\mathrm{NV}+\mathrm{RP}$ & $<0.001^{*}$ & $<0.001^{*}$ & $<0.001^{*}$ & $<0.001^{*}$ & $<0.001^{*}$ & & \\
\hline $\mathrm{DT}+\mathrm{RP}$ & 0.296 & 1.000 & 1.000 & 1.000 & 1.000 & $0.008^{*}$ & \\
\hline $\mathrm{DT}+\mathrm{NV}+\mathrm{RP}$ & $<0.001^{*}$ & $<0.001^{*}$ & $<0.001^{*}$ & $<0.001 *$ & $<0.001 *$ & 1.000 & $<0.001^{*}$ \\
\hline
\end{tabular}

*Significant $(p<0.05) \mathrm{N}=$ Normal, NV= No Vision, RP= Reduced Proprioception, NV+RP= No Vision + Reduced Proprioception, DTC, Dual-Task Costs; ML, Medial-Lateral; RMS, root mean square; AP, anterior-posterior; $\mathrm{V}$, average speed of displacement; AoE, area of the 95 th percentile ellipse. 
Table 5. Postural Dual Task Costs (DTCp) Change Between All Test Situations in All, Fallers and Non-Fallers

\begin{tabular}{|c|c|c|c|c|c|c|c|}
\hline \multicolumn{8}{|c|}{ All } \\
\hline Max-ML & 67 & 35 & 0.342 & 23 & 0.088 & 14 & $0.040^{*}$ \\
\hline RMS-ML & 58 & 26 & 0.237 & 28 & 0.201 & 12 & $0.049^{*}$ \\
\hline Max-AP & 46 & 40 & 0.987 & 27 & 0.918 & -1 & $0.008^{*}$ \\
\hline \multicolumn{8}{|c|}{ Fallers } \\
\hline Max-ML & 37 & 8 & 0.35 & 31 & 0.88 & 21 & 0.63 \\
\hline RMS-ML & 35 & 12 & 0.49 & 37 & 0.94 & 16 & 0.57 \\
\hline Max-AP & 40 & 45 & 0.92 & 23 & 0.64 & -2 & 0.61 \\
\hline \multicolumn{8}{|c|}{ Non-Fallers } \\
\hline Max-ML & 88 & 54 & 0.37 & 17 & 0.06 & 9 & $0.04 *$ \\
\hline RMS-ML & 74 & 37 & 0.16 & 21 & $0.05 *$ & 9 & $0.02 *$ \\
\hline Max-AP & 49 & 45 & 0.87 & 31 & 0.48 & 0 & 0.06 \\
\hline RMS-AP & 42 & 31 & 0.61 & 30 & 0.55 & 2 & 0.07 \\
\hline AoE & 179 & 106 & 0.35 & 68 & 0.15 & 12 & $0.04 *$ \\
\hline
\end{tabular}

$\mathrm{N}=$ Normal, NV= No Vision, RP= Reduced Proprioception, NVRP= No Vision + Reduced Proprioception, DTC, dual-task costs; ML, medial-lateral; SD, standard deviation; RMS, root mean square; AP, anterior-posterior; V, average speed of displacement; AoE, area of the 95th percentile ellipse. *Significant $(p<0.05)$ difference between "normal situation."

As expected, fallers showed more disturbance of their postural control when vision, somatosensory information or the combination of vision and somatosensory information where reduced. Our data showed that with an increasing reduction of sensory information, fallers and non-fallers reacted differently. Non-fallers had a close to no reaction affecting postural control due to the additional task imposed in the reduced sensory information situation. This reaction can be inferred from the DTCp. Non-Fallers reduced their DTCp significantly whereas the fallers maintained their DTCp on a constant level. It can be speculated from this that non-fallers experience no threats when the stability of their postural control is challenged in this combined reduced sensory situation. The small reduction of postural control leaves enough resources for a steady cognitive tasks performance. These results could be explained by the adaptive resource-sharing model [9]. Fallers showed a reduction of postural control in the combined reduced sensory situation caused by the additional task. There must have been a transfer of resources. But we are not sure where these resources come from, because there was steady cognitive tasks performance. One explanation could be a direct transfer of recourses from the postural control to the additional task. An alternative explanation could be that we measured cognitive performance accuracy and not counting speed. It is possible that the fallers just counted slower and, therefore, had more resources left available for their postural stability. In this case both tasks deteriorated and the transfer of resources could be supportive for the theoretical framework of resource-sharing. One or both performances may deteriorate if they require more than the available attentional resources $[10,11]$. Future study should focus on a more precise cognitive performance assessment to test this assumption.

A limitation of the study was the relatively small sample size. For this reason, the findings cannot be generalized to the broader community based on this study alone. The findings warrant, however, further research in larger samples.

\section{CONCLUSION}

This study was designed to observe postural control under reduced vision and reduced somatosensory information and in a combination of these two. The combination of reduced visual and somatosensory information caused larger disturbance of postural control in fallers. Offering an additional task apparently causes postural control in fallers to decrease. These results have potential clinical relevance in settings where training of postural control is offered to populations at risk, e.g. in fall prevention programs for the elderly. The findings are, therefore, a good starting point for further research examining the effects of different exercises in elderly to reduce the risk of falling. It can for example be speculated that so-called dual task forms of exercise in a reduced sensory situation, and that would more mimic a "real life" situation, represent one of the necessary stages in a progressive balance training program that takes principles of training in to account [22]. 


\section{ACKNOWLEDGMENT}

The authors wish to thank Anna Burini for performing the measurements.

\section{COMPETING INTERESTS}

The authors declare that they have no competing interests.

\section{AUTHORS' CONTRIBUTIONS}

JS designed and performed the study and wrote the manuscript. EDB helped to draft the manuscript, monitored the study, and revised the manuscript critically for its content. SH monitored and revised the manuscript critically for its content. DU helped with the coordination. TM revised the manuscript and initiated the study. All authors read and approved the final manuscript.

\section{REFERENCES}

[1] Goble DJ, Coxon JP, Wenderoth N, Van Impe A, Swinnen SP. Proprioceptive sensibility in the elderly: degeneration, functional consequences and plastic-adaptive processes. Neurosci Biobehav Rev 2009; 33: 271-8.

[2] Fitzpatrick R, Rogers DK, McCloskey DI. Stable human standing with lower-limb muscle afferents providing the only sensory input. J Physiol 1994; 480: 395-403.

[3] Kerr B, Condon SM, McDonald LA. Cognitive spatial processing and the regulation of posture. J Exp Psychol Hum Percept Perform 1985; 11: 617-22.

[4] Mulder T, Zijlstra W, Geurts A. Assessment of motor recovery and decline. Gait Posture 2002; 16: 198-210.

[5] Melzer I, Benjuya N, Kaplanski J. Age-related changes of postural control: Effect of cognitive tasks. Gerontology 2001; 47: 189-94.

[6] Lundin-Olsson L, Nyberg L, Gustafson Y. 'Stops walking when talking' as a predictor of falls in elderly people. Lancet 1997; 349: 617.

[7] Teasdale N, Bard C, LaRue J, Fleury M. On the cognitive penetrability of postural control. Exp Aging Res 1992; 19: 1-13.

[8] Doumas M, Smolders C, Krampe RT. Task prioritization in aging: Effects of sensory information on concurrent posture and memory performance. Exp Brain Res 2008; 187: 275-81.
Mitra S. Adaptive utilization of optical variables during postural and suprapostural dual-task performance: Comment on Stoffregen, Smart, Bardy, and Pagulayan (1999). J Exp Psychol Hum Percept Perform 2004; 30: 28-38.

[10] Woollacott M, Shumway-Cook A. Attention and the control of posture and gait: A review of an emerging area of research. Gait Posture 2002;16: 1-14.

[11] Shumway-Cook A, Woollacott M. Attentional demands and postural control. the effect of sensory context. J Gerontol A Biol Sci Med Sci 2000; 55(1): 10-6.

[12] Swanenburg J, de Bruin ED, Uebelhart D, Mulder T. Compromising postural balance in the elderly. Gerontology 2009; 55: 353-60.

[13] Pajala S, Era P, Koskenvuo M, Kaprio J, Tolvanen A, Rantanen T. Genetic and environmental contribution to postural balance of older women in single and dual task situations. Neurobiol Aging 2007; 28: 947-54.

[14] Pellecchia GL. Dual-task training reduces impact of cognitive task on postural sway. J Mot Behav 2005; 37: 239-46.

[15] Lezak MD. Neuropsychological Assessment, 4th Ed. New York, Oxford University Press; 2004.

[16] Swanenburg J, de Bruin ED, Favero K, Uebelhart D, Mulder T. The reliability of postural balance measures in single and dual tasking in elderly fallers and non-fallers. BMC Musculoskeletal Disord 2008; 9: 162 .

[17] Gibson MJ, Andres RO, Isaacs B, Radebaugh T, Worm-Petersen J. The prevention of falls in later life. A report of the Kelloggs International Work Group. Danish Med Bull 1987; 34: 1-24.

[18] Buchner DM, Hornbrook MC, Kutner NG, et al. Development of the common data base for the FICSIT trials. J Am Geriatr Soc 1993; 41: 297-330.

[19] Corriveau H, Hebert R, Prince F, Raiche M. Postural control in the elderly: An analysis of test-retest and interrater reliability of the COP-COM variable. Arch Phys Med Rehabil 2001; 82: 80-5.

[20] Cohen J. Statistical Power Analysis for the Behavioral Sciences, 2nd Ed. New York, Lawrence Erlbaum Assoc 1988.

[21] Cockburn J, Haggard P, Cock J, Fordham C. Changing patterns of cognitive-motor interference (CMI) over time during recovery from stroke. Clin Rehabil 2003; 17: 167-73.

[22] Mansfield A, Peters AL, Liu BA, Maki BE. A perturbation-based balance training program for older adults: study protocol for a randomised controlled trial. BMC Geriatr 2007; 31: 7-12. 